Bäckström u. Paijkull: Volum u. Kohlenstoffgehalt der bei der Auflösung etc. 683

\title{
Volum und Kohlenstoffgehalt der bei der Auflösung von Eisen in Säuren entwickelten Gasarten.
}

\author{
Von \\ Helge Bäckström und Gunnar Paijkull*) \\ in Stockholm.
}

(Mittheilung aus dem Stockholmer Universitäts-Laboratorium.)

Torbern Bergman**) war, so weit uns bekannt, der Erste, welcher das Volum der bei der Auflösung von Eisen in Säuren entwickelten Gase maass. Er fand, dass $\gg$ Schwefelsäure und Salzsäure gleiche Volumina ,brennbares Gas' aus gleichen Gewichtsmengen desselben Eisens éntwickelten, doch brauchte die erste Säure längere Zeit zur Auflösung als die zweite $\ll$. $\gg$ Die Art und Darstellungsweise des Eisens haben dagegen eine sehr grosse Einwirkung auf das entwickelte Gasvolnm. — $-\gg$ Die Grenzwerthe sind, auf $1 \mathrm{~g}$ Substanz berechnet, für

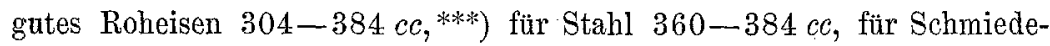
eisen 384-408 cc. Roheisen von derselben Hütte gibt um so weniger Gas, je weisser es ist. «

Gleichzeitig and mit Bergman's Resultaten übereinstimmend ist Hjelm's Untersuchung; $\dagger$ ) er wies auch den Kohlenstoffgehalt der Gase nach.

Die französischen Forscher Vandermonde, Berthollet und Monge $\uparrow$ ) haben auch Volummessungen dieser Gase angestellt. Die Verschiedenheit zwischen dem von Stahl und Schmiedeeisen entwickelten Gasvolum suchen sie ganz richtig in einer Verschiedenheit des Kohlenstoffgehaltes; da aber weisses Roheisen, worin sie keinen merkbaren Kohlenstoffgehalt vermuthen (!), gerade die Eisensorte ist, welche das kleinste Gasvolum liefert, stellen sie die irrthümliche Behauptung auf, dass Roheisen, und besonders weisses, Sauerstoff enthält, weswegen es

*) Die Originalabhandlung wurde der schwedischen Academie der Wissenschaften am 9. März 1887 vorgelegt.

**) „De analysi ferri." Stockholm 1781. von uns.

***) Die Reductionen auf die üblichen Maass- und Gewichtseinheiten sind

†) Nur im Mannscript vorbanden.

†) Mémoire de l'Acad. des Sciences 1786. 
bei der Auflösung nicht so viel Wasser zu zersetzen braucht, als reines Eisen, und also nicht so viel Wasserstoff liefern kann. Diese unrichtige Voraussetzung wird von Karsten*) gründlich widerlegt. - Ausser einer Volummessung von Berzelius ist später, so weit uns bekannt, nur eine Untersuchung über die Natur der entwickelten Gase von $\mathrm{Hahn} * *$ ) ausgeführt worden.

Der von uns bei der Mehrzahl der Analysen benutzte Apparat ist in Fig. 49 abgebildet. Er besteht aus einem Probirrohr ohne ausgebogene Kante, auf welches durch ein weites Stück Gummischlauch ein Oberstz̈ck von derselben Weite wie das Probirrohr befestigt ist. Dieses ist oben ebenfalls durch Gummischlauch mit einem graduirten Rohr verbunden, welches mit Wasser umgeben werden kann, und steht vermittelst eines Seitenrohres und eines ziemlich Iangen Kautschukschlauches mit einer verdünnte Schwefel-oder Salzsäure enthaltenden Kochflasche in Verbindung. - Nachdem die von der Säure absorbirte Luft ausgekocht ist, wird etwas Säure in das Probirrohr eingesaugt, um die Fig. 49.

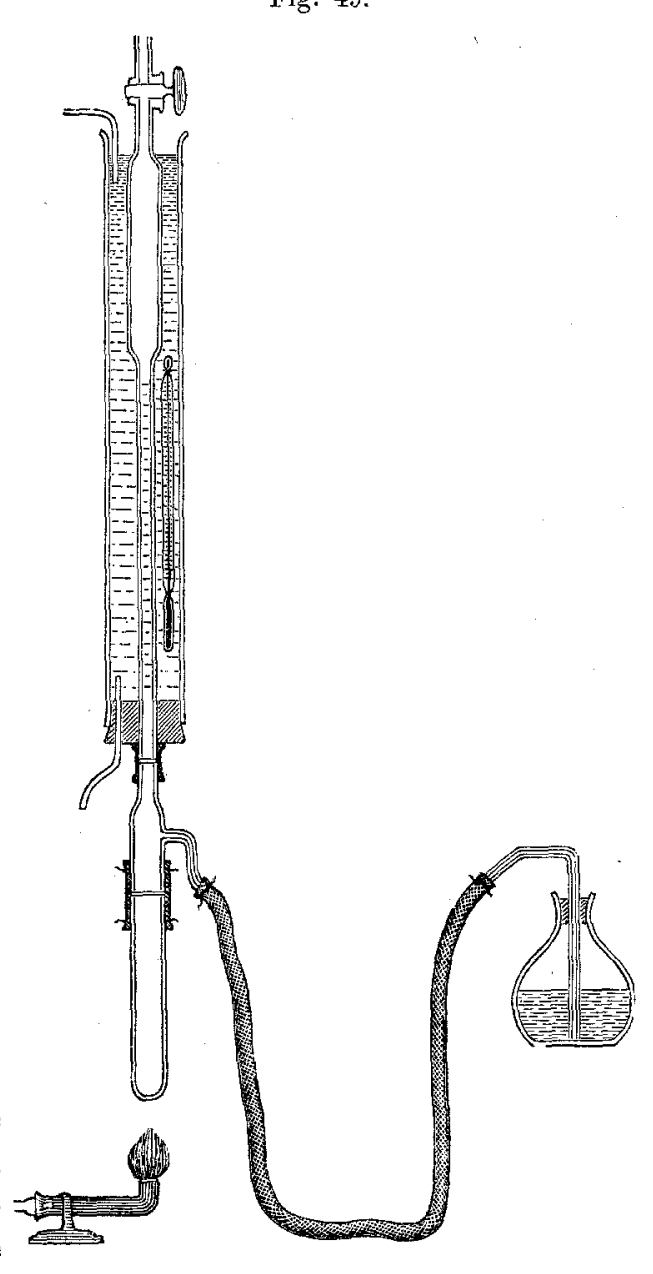
Leitungen zu füllen; die Kochflasche wird darnach gehoben, der Ap-

*) „Handbuch der Eisenhüttenkunde."

2. Aufl. Berlin 1827. S. 255.

**) Ann. Chem. Pharm. 129, 57. 
parat mit der heissen, verdünnten Säure bis zum Glashabne gefüllt und durch Zudrehen desselben verschlossen. - Die Kochflasche wird etwas gesenkt, ein Gasbrenner unter die Röhre gesetzt und das Kochen noch eine Weile fortgesetzt, um die letzten Spuren von Luft durch Kochen unter vermindertem Luftdrucke auszutreiben. (Das graduirte Rohr ist während dieser Operationen nicht von Wasser umgeben.) Das gebildete kleine Luftvolum wird auf beschriebene Weise ausgetrieben, und indem der Hahn fortwährend offen gehalten wird, lässt man die zu untersuchende Eisenprobe, welche in unseren Versuchen nur 0,1 bis $0,2 g$ beträgt, binuntergleiten; der Hahn wird schnell verschlossen, die Kochflasche ein wenig gesenkt and die Auflösung fängt an, durch mässige Erwärmung unterstützt.

Wenn die Gasentwickelung aufhört, wird der Gasbrenner weggenommen und das aufgesammelte Gasvolum zu constanter Temperatur abgekuthlt, indem man einen Wasserstrom durch das Mantelrohr leitet. - Nach einiger Zeit, wenn das Volum constant geworden ist, wird abgelesen mit den beiden Wasserflächen in gleicher Höhe. - Durch Heben der Kochflasche kann später das Gas mit Hülfe eines kleinen umgebogenen Glasröhrchens, welches mittelst eines Gummischlauches an dem Hahnrohr fest angesetzt wird, in den Gasanalysenapparat eingetrieben werden. Bei allen unseren Versuchen diente Professor 0. Pettersson's Apparat, beschrieben in dieser Zeitschrift 1886, Heft 4.

Da die Lösung ohne Luftzutritt vor sich geht, hatten wir diese Experimente mit Titrirungsbestimmung des Eisens durch Kaliumpermanganat $z u$ verbinden gedacht. Die erhaltenen Resultate waren jedoch schwankend und gewöhnlich zu hoch, was wir dadurch erklären, dass ein Theil des Kohlenstoffes sich in der Flüssigkeit gelöst befindet in Form irgend einer organischen Verbindung, die reducirend wirkt und einen eigenthümlichen Geruch hat. Diese Vermuthung wird dadurch bestätigt, dass der Geruch während der Titrirung verschwand, sogar früher als Färbung eintrat.

Folgende Tabelle enthält eine Zusammenstellung unserer Resultate: 


\begin{tabular}{|c|c|c|c|}
\hline $\begin{array}{c}\text { Kohlen- } \\
\text { stoff } \\
\text { im Eisen } \\
\text { (total) } \\
0 / 0\end{array}$ & eisensore & $\begin{array}{l}c c \text { von } \\
1 g \mathrm{Fe}\end{array}$ & $\begin{array}{c}\text { Kohlen- } \\
\text { stoff } \\
\text { im Gas } \\
0 / 0\end{array}$ \\
\hline 0,00 & Reines Eisen (Atomgew. 55,91) soll entwickeln: & 399,6 & - \\
\hline 0,11 & Eggertz Normaldraht....... & 398,7 & - \\
\hline 0,20 & Eisendraht. . . . . . . . . . . . & 396,9 & - \\
\hline 0,26 & Eggertz Normaldraht . . . . . & $\begin{array}{l}392,5 \\
393,6\end{array}$ & $\begin{array}{l}0,180 \\
0,165\end{array}$ \\
\hline 0,50 & Eisendraht von Bofors. . . . . . . & 378,5 & 0,346 \\
\hline 0,60 & $n \quad n \quad n \quad . . \cdot \cdot \cdot \cdot \cdot$ & 386,3 & 0,356 \\
\hline 0,60 & Eisen . . . . . . . . . . & 391,3 & - \\
\hline 1,00 & Stahldrahtvon Bofors. . . . .. & 356,0 & 0,242 \\
\hline 1,00 & Bessemerstahl mit $0,13 \%$ Graphit. . & $\begin{array}{l}383,2 \\
380,2\end{array}$ & $\begin{array}{l}0,776 \\
0,645\end{array}$ \\
\hline 1,30 & Eggertz Normaldraht . . . . . . . & 353,3 & 0,492 \\
\hline 3,28 & Weisses Roheisen mit $0,41 \%$ Graphit. & $\begin{array}{l}297,8 \\
298,4\end{array}$ & $\begin{array}{l}1,94 \\
2,12\end{array}$ \\
\hline 3,80 & Graues Roheisen mit $3,45 \%$ Graphit. . & 383,3 & - \\
\hline 3,87 & $\begin{array}{l}\text { Weisses Roheisen: } \\
\text { gelöst in Schwefelsäure }\end{array}$ & $\begin{array}{l}295,7 \\
290,8 \\
282,8 \\
309,5 \\
311,1\end{array}$ & $\begin{array}{l}2,245 \\
2,276 \\
2,235 \\
2,546 \\
2,552\end{array}$ \\
\hline 3,90 & Graues Roheisen mit $3,55 \%$ Graphit. . & 372,0 & $\therefore$ \\
\hline 4,24 & Weisses Roheisen * * * & 296,3 & 2,806 \\
\hline $0, a d$ & 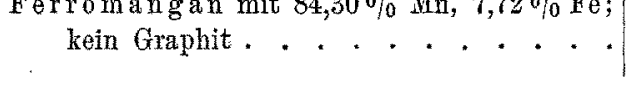 & 287,5 & 3,80 \\
\hline
\end{tabular}

Die von uns gefundenen Gasvolumina liegen innerhalb der schon von Bergman bestimmten Grenzen. Unsere Bestimmungen des Kohlenstoffgehaltes bestätigen die von Berzelius gemachte Wahrnehmung, dass nicht aller gebundene Kohlenstoff in dem entweichenden Gase enthalten ist; unsere Analysen zeigen anch, dass derjenige Theil des Kohlenstoffes, der als Kohlenwasserstoffgas entweicht, dem Gebalt an gebundenem Kohlenstoff nicht proportional is t. Quantitative Bestimmungsmethoden, bei welchen man den im Eisen chemisch gebundenen Kohlenstoff durch Analyse der mittelst verdünnter Säuren entwickelten Gase bestimmt, sind deswegen zu verwerfen, da es feststeht, dass keine von uns untersuchte Eisensorte weder ihren ganzen Gehalt an gebundenem Kohlenstoff, 
noch einen proportionalen Theil desselben als Gas abgegeben hat.

Die Bergman'sche Wahrnehmung, dass Schwefelsäure und Salzsäure gleiche Mengen von Gas entwickeln, scheint bei hohem Procentgehalt an chemisch gebundenem Kohlenstoff nicht zuzutreffen, wenigstens zeigen die mit besonderer Rücksicht hierauf ausgeführten Versuche (weisses Roheisen mit $3,87 \%$ C) ganz bestimmt, dass Salzsäure grössere Volumina und mehrKohlenstoff im Gas lieferte als relativ gleich starke Schwefelsäure (1:25).

Aus unseren Resultaten geht ferner hervor, dass der in der Flüssigkeit zurückbleibende Theil des Kohlenstoffes grosse Wasserstoffquantitäten mit sich vereinigt hält, was eine beträchtliche Verminderung des gebildeten Gasvolums hervorruft. Wie schon für den als Gas entweichenden Kohlenstoffgehalt gezeigt, ist auch das entwickelte Gasvolum mit dem Gehalt an chemisch gebundenem Kohlenstoff nicht proportional. Approximativ kann man im allgemeinen von einem grösseren Gasvolum auf einen kleineren Kohlenstoffgehalt schliessen, aber nicht quantitativ.

Man hat sich schon lange bemüht, das ungleiche Verhalten von gehärtetem and ungehärtetem Stahl bei der Auflösung in Säuren auf eine Verschiedenheit in der Bindungsweise zwischen dem Kohlenstoff und dem Eisen zurückzuführen. Die wichtige Beobachtung, dass gehärteter Stahl beim Auflösen einen geringeren Rückstand liefert als ungehärteter, findet man zuerst in Karsten's Arbeit.*) $\mathrm{Zu}$ demselben Resultat kam Caron, ${ }^{* *}$ ) welcher dazu noch bemerlkt, dass kalt bearbeiteter oder gehämmerter Stahl sich in dieser Hinsicht ähnlich dem gehärteten verhält. $\mathrm{R}$ inman ${ }^{* * *}$ ) fand, dass gehärteter Stahl beim Lösen in Chlorwasserstoffsäure keinen kohlenstoffhaltigen Rückstand gibt, bei ungehärtetem Stahl dagegen fand er einen aus fein vertheilten Kohlenstoffpartikeln bestehenden Rückstand, der jedoch bei fortgesetztem Kochen verschwinden konnte und also nicht Graphit war. Aus diesen Beobachtungen schloss er, dass ausser Graphit zwei ver-

*) l. c. $\$ 216-217$.

**) Comptes rendus 1863 , S. 43 u. 210.

***) Debers. der Verhandl. der schwed. Acad. d. Wiss. 1865, s. 443. 
schiedene Arten von chemisch gebundenem Kohlenstoff im Stahl vorhanden sind, die er Cement- und Härtungs-Kohlenst off nannte.

Die neuesten Forschungen haben zu interessanten Schlussfolgerungen über das Vorkommen des Kohlenstoffes im Stahl geführt. Darnach existirt in demselben eine Verbindung von Eisen mit Kohle - ein Carburet - mit noch nicht endgültig festgestellter Formel, welches im Stahl eine Art von Zellengewebe ( $\$$ réseau «) bildet, dessen Maschen oder Zwischenräume von reinem Eisen ausgefüllt sind. Nach Osmond's und Werth's*) Ansicht ist der sogenannte Cement-Kohlenstoff ( $\gg$ carbone de recuit $«$ ) mit Eisen zu diesem Carburet verbunden, der sogenannte Härtungs-Kohlenst off ( $\gg$ carbone de trempe $\ll$ ) sollte sich dagegen nicht in chemischer Verbindung, sondern in dem Eisen aufgelöst befinden, welches die Hauptmasse des Stahls bildet (disséminé dans les noyeaux cellulaires). Beim Auflösen sollte der HärtungsKohlenstoff zum grösseren oder kleineren Theil in Form gasförmiger Producte weggehen, während der Cement-Kohlenstoff als Carburet zum grösseren oder kleineren Theil ungelöst zurückbleibt.

Ohne näher auf diese Frage einzugehen, ist die folgende Untêrsuchung angestellt worden, um den Einfluss der Härtung oder der mechanischen Bearbeitung in der Kälte auf die Menge des bei der Auflösung des Stahls in gasförmige Verbindungen übergehenden Kohlenstoffs zu studiren. Sämmtliche oben erwähnten Autoren haben sich nämlich hauptsächlich mit den hinterbleibenden Rückständen beschäftigt. Wir dachten deswegen, dass eine Untersuchung der beim Auflösen entwickelten gasförmigen Producte die Kenntniss des Verhältnisses zwischen den verschiedenen Kohlenstoffarten des Eisens vervollständigen und erweitern sollte. Einer von uns, G. Paijkull, hat daher diese Untersuchung vorgenommen.

Es ergibt sich aus derselben, dass gehärteter Stahl ein grösseres Volum von einem an Kohlenstoff reicheren Gas gibt als ungehärteter.

Die folgende Tabelle enthält die Resultate:

*) Ann. des mines 1881, VIII, S. 8. 


\begin{tabular}{|c|c|c|c|c|c|c|}
\hline \multirow[b]{2}{*}{$\begin{array}{l}\text { Ana- } \\
\text { lyse }\end{array}$} & \multirow[b]{2}{*}{ Eisensorte } & \multirow{2}{*}{$\begin{array}{c}\text { Kohlen- } \\
\text { stoff- } \\
\text { gehalt } \\
\text { in } 0 / 0\end{array}$} & \multicolumn{2}{|c|}{ Ungehärtet } & \multicolumn{2}{|c|}{ Gehärtet } \\
\hline & & & $\begin{array}{l}\text { cc Gas } \\
\text { aus } \\
1 g \mathrm{Fe}\end{array}$ & $\begin{array}{ccc}g & \mathrm{C} & \mathrm{im} \\
\mathrm{Gas} & \text { aus } \\
1 & g & \mathrm{Fe}\end{array}$ & $\begin{array}{l}c c \text { Gas } \\
\text { aus } \\
1 g \mathrm{Fe}\end{array}$ & $\begin{array}{l}g \mathrm{C} \text { im } \\
\text { Gas aus } \\
1 g \text { Fe }\end{array}$ \\
\hline I 1. & Eisendraht von Bofors & 0,30 & 393,8 & 0,0009 & 398,5 & 0,0018 \\
\hline 2. & $\Rightarrow \quad n \quad n$ & $\eta$ & $n$ & 0,0013 & $"$ & 0,0018 \\
\hline II 1. & " & 0,50 & 384,9 & 0,0015 & 397,7 & 0,0035 \\
\hline 2. & $" \quad " \quad "$ & \% & - & - & $n$ & 0,0034 \\
\hline 3. & $\begin{array}{l}\text { Eisendraht von Bofors, } \\
\text { kalt gehämmert }\end{array}$ & " & 380.4 & 0,0012 & - & - \\
\hline 4 . & $\begin{array}{c}\text { Eisendraht von Bofors, } \\
\text { kalt gehämmert }\end{array}$ & " & $"$ & 0,0013 & - & - \\
\hline III 1. & Eisendraht von Bofors & 0,60 & 386,3 & 0,0034 & 395,0 & 0,0045 \\
\hline 2 & $n \quad " \quad n$ & $"$ & $"$ & 0,0038 & $\eta$ & 0,0044 \\
\hline IV 1. & " & 1,00 & 356,0 & 0,0025 & 386,1 & 0,0088 \\
\hline 2. & " & " & 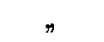 & 0,0023 & " & 0,0077 \\
\hline 3. & $n$ & " & 354,0 & 0,0016 & 386,1 & 0,0088 \\
\hline 4. & $* \quad \Rightarrow \quad m$ & ” & 355,6 & - & - & - \\
\hline 5 . & $\begin{array}{l}\text { Eisendraht von Bofors, } \\
\text { kalt gehümmert }\end{array}$ & $"$ & 345,1 & 0,0012 & - & 一 \\
\hline 6 . & $\begin{array}{l}\text { Eisendraht von Bofors, } \\
\text { kalt ge eämmert }\end{array}$ & $y$ & 345,4 & 0,0013 & - & - \\
\hline V 1. & Eggertz Normaldraht & 1,30 & 359,2 & 0,0051 & 383,4 & 0,0083 \\
\hline 2. & $"$ & $و$ & 353,3 & - & 384,3 & - \\
\hline 3. & $"$ & y & 351,2 & 0,0044 & - & - \\
\hline
\end{tabular}

Der Einfluss der Härtang ist also sehr bedeutend: Dieselbe Stahlsorte von $1 \%$ Kohlenstoffgehalt, welche in ungehärtetem Zustand $356 \mathrm{cc}$ (mit 2,5 mg C) gab, entwickelte nach der Härtung 386,1 cc (mit $8,8 \mathrm{mg} \mathrm{C}$ ), kalt gehämmerter aber nur $345,1 c c$ (worin $1,2 \mathrm{mg} \mathrm{C}$ ). Wie zum Beispiel aus den Analysen IV ersichtlich, scheint es fast, als ob der sogenaunte Härtangs-Kohlenstoff vollständig oder fast vollständig in Gasform übergehe; ob aber nicht auch etwas ron dem sogenannten Cement-Kohlenstoff in dem Gase vorhanden ist, ist schwer za beurtheilen.

Caron fand, wie erwähnt, dass mechanische Bearbeitung, wie Hämmern in der Kälte etc., auf den Stahl gewissermaassen härtend einwirkte; $0 \mathrm{smond}$ and $\mathrm{Werth}$ fanden dies jedoch nicht bestätigt, sondern behaupten, dass mechanische Bearbeitung ohne Temperaturerhöhung ohne Einfluss auf den Kohlenstoff im Stahle ist; unsere Analysen zeigen, dass der kalt gehämmerte Stahl (siehe Analyse II 3, 4 und IV 5, 6), ein kleineres Gasvolum und weniger Kohlenstoff im Gas ergab als die ursprüngliche Probe. 